Research Article

\title{
Boosting Antimicrobial Activity of Imipenem in Combination with Silver Nanoparticles towards $S$. fonticola and Pantoea sp.
}

\author{
Shaimaa Obaid Hasson ${ }^{1}$, Mohammed Jabber Al-Awady² ${ }^{2}$ Adnan Hamad Al-Hamadani ${ }^{3}$, Ibtisam \\ Habeeb Al-Azawi ${ }^{3}$, Alaa Irhayyim $\mathrm{Ali}^{3}$ \\ ${ }^{1}$ Department of Microbiology, College of Veterinary, Al-Qasim Green University, Iraq. \\ ${ }^{2}$ Department of Genetic Engineering, Faculty of Biotechnology, Al Qasim Green University, Babylon, Iraq. \\ ${ }^{3}$ Department of Medical Microbiology, College of Medicine, Al-Qadisiyah University, Iraq. \\ $\simeq$ Corresponding author. E-mail: shaimaaobead@gmail.com.
}

Received: Mar. 28, 2019; Accepted: Jun. II, 2019; Published: Jun. 20, 2019.

Citation: Shaimaa Obaid Hasson, Mohammed Jabber Al-Awady, Adnan Hamad Al-Hamadani, Ibtisam Habeeb Al-Azawi, and Alaa Irhayyim Ali, Boosting Antimicrobial Activity of Imipenem in Combination with Silver Nanoparticles towards S. fonticola and Pantoea sp. Nano Biomed. Eng., 20I9, II(2): 200-2I4.

DOI: $10.5101 /$ nbe.v11i2.p200-214.

\begin{abstract}
Silver nanoparticles have been considered as powerful antimicrobial agents recently, especially with the increasing incidence of diseases associated with biofilm and multi-drug resistant pathogens. The aim of this study was to synthesize silver nanoparticles by biological and chemical methods and combination with imipenem to eradicate biofilm-forming bacteria at phenotypic and genotypic levels. The biosynthesis of silver nanoparticles was done by using Enterobacter cloacae (cell-free suspension) while chemosynthesis was conducted using sodium borohydride. Biological and chemical silver nanoparticles were characterized by ultraviolet-visible spectrophotometry which showed absorbance peak at 400 and 390nm respectively. Fourier transformer infrared analysis revealed that carboxylic and polyphenolic groups were coated on surface of both silver nanoparticles. Scanning electron microscope and size analyser showed that the sizes of biologically and chemically silver nanoparticles were $63 \mathrm{~nm}$ and $25 \mathrm{~nm}$, respectively. In addition, it showed the formation of cubical nanoparticles. The antimicrobial effect of synthesized silver nanoparticles were evaluated by agar well diffusion and macrodilution method to determine minimum inhibitory concentration value. The results showed that biological silver nanoparticles were more effective on biofilm forming bacteria (Serratia fonticola and Pantoea sp.) than chemical synthesized ones. In addition, the combination effect between silver nanoparticles and imipenem displayed synergistic effect. Gene expression of biofilm encoding genes (smaI and esaL) were evaluated by real- time quantitative polymerase chain reaction (RT- qPCR) before and after treatment with silver nanoparticles in both types and imipenem and in combination between them. The results revealed that biological silver nanoparticles alone or in combination with antibiotics were more effective on biofilm gene expression by down regulation than other treatments.
\end{abstract}

$\overline{\text { Keywords: Biofilm; Silver nanoparticles; Serratia fonticola; Pantoea sp.; Enterobacter cloacae; }}$ Imipenem

\section{Introduction}

Silver ions are considered to have antimicrobial properties. In the early nineteenth century, $0.5 \%$ silver nitrate was utilized for the treatment and prevention of microbial infections such as Ophthalmia neonatorum. 
The use of silver slowly lessened, when penicillin antibiotic was discovered [1]. Currently, the utilization of silver has become important for treating infections because of the lack of efficacy of conventional drugs. However, silver ion is easily inactivated by complexation and precipitation. Consequently, the use of silver ions is imperfect [2]. Nano silvers, which are zero valent, can be a valued alternative to silver ions [3]. Nanoparticles (NPs) are clusters of atoms, with sizes ranging from 1 to $100 \mathrm{~nm}$, whereas a "nano" is used to designate one billionth of a meter [4]. Due to the fact that their small particle size and high surface area, silver NPs have unique physical and chemical properties which are different from those of metallic silver.

Silver NPs are a non-toxic and safe antibacterial agent for the human body in addition to its procession of an antifungal activity [5], anti-inflammatory properties [6], antiviral activity [7], anti-angiogenic activity [8] and anticancer activity. Nano silver has been utilized in the functionalization of materials in order to improve the performance of clothing, semiconductor, and nanocomposite materials. For example, in the manufacturing silver NPs coated medical devices for infection-free transplantation [9, 10]. Silver NPs have also shown a synergistic activity in combination with antibiotics for example, a combination of amoxicillin and nano silver demonstrated superior bactericidal efficiency towards Escherichia coli than when they were separately applied [11], and interactions between silver NPs. and polymyxin B revealed synergistic effects against gram negative bacteria [12]. Hwang et al. also reported the synergistic antibacterial activity of nano silver in combination with the conventional antibiotics ampicillin, chloramphenicol and kanamycin against various pathogenic bacteria. The study illustrated that nano-Ags had potential as a combination therapeutic agent for the treatment of infectious diseases by bacteria [13].

Recently, some bacteria have developed themselves to be resistant towards the commonest antibiotics. They have the ability to stay alive and even reproduce in the presence of an antibiotic. The biofilm production is also associated to the antibiotic resistance problem [14]. Bacteria are used to adhere to surfaces through a biofilm matrix, a 3D, gel-like, highly hydrated and locally charged environment. Adhesion of these bacteria to other organs may contribute to the pathogenesis of infection [15]. For instance, S. typhi has demonstrated resistance towards chloramphenicol, ampicillin, quinolone, and trimethoprim, and E. coli showed resistance towards ampicillin, kanamycin, sulfisoxazole, streptomycin, tetracycline, ticarcillin, and so on [16, 17]. Al-Azawi et al. studied the formation of biofilm in terms of the utilization of icaA genes presented in $S$. lentus, responsible for biofilm formation [18]. In addition to that, Hasson et al. achieved the same study on the basis of esaI and smaI genes present in Pantoea sp. and $S$. fonticola, respectively [19]. These genes could be considered as the indicator of biofilm formation in urinary catheterized patients, which in turn was considered as a serious reason of multi-drug resistance in bacteria. Thus, nanoparticle-based antibacterial formulations could be effective bactericidal materials owing to the combined effects of silver NPs and antibacterial agents.

Herein, in the present study, we synthesized silver NPs according to the biosynthesis and chemical method. Then, we investigated the antibacterial activity of green synthesized and chemically synthesized silver NPs alone and in combination with imipenem as an antibiotic using well diffusion agar and macrodilution method to determine MIC value against bacteria (Serratia fonticola and Pantoea sp.). The Gene expression of biofilm encoding genes encoding (smaI and esaL) were evaluated by real- time quantitative polymerase chain reaction (RT- qPCR) before and after treated with green and chemical synthesized silver NPs alone and combined with imipenem.

\section{Experimental Materials}

Imipenem (500 mg vial) was purchased from Merck (USA). Azithromycin $(200 \mathrm{mg} / 5 \mathrm{~mL})$ was purchased from Reva Phrma (Egypt). Silver nitrate was provided from Sigma Aldrich (USA). Congo red stain, ethidium bromide, Kovac's reagent, and methyl red were purchased from Sigma Aldrich (USA). Deionized water and DNA marker ladder were supplied from Pioneer (Korea). The following chemicals were used in the preparation of culture media: agar, blood agar, brain heart infusion agar, brain heart infusion broth, carbohydrate fermentation media, eosin methylene blue, MacConkey agar, Muller Hinton agar, Muller Hinton broth, nutrient agar, nutrient broth, and trypticase soy broth were all purchased from Himedia (India). Gram stain was provided by BDH. (UK). This solution is often used for studying the 
cell morphology and arrangement of bacterial cells. Phosphate buffer saline (PBS) was prepared through dissolving one tablet of PBS (pH 7.3) in $100 \mathrm{~mL}$ of D.W to prepare this solution; the resulting solution was sterilized by autoclave. $1 \times$ tris borate EDTA (TBE) buffer was prepared by diluting the concentrated TBE buffer $(10 \times)$. It was used to dissolve agarose and in electrophoresis procedure. $100 \mathrm{~mL}$ of TBE $(10$ $\times$ ) was added to $900 \mathrm{~mL}$ of D.W to reach to $1 \times \mathrm{TBE}$ concentration. All other reagents were purchased from $\mathrm{BDH}$, UK and are of the highest commercial grade available. Highly purified water was used everywhere in the experiments with analytical grade.

\section{Synthesis of silver nanoparticles}

The synthesis of silver NPs were conducted according to reference [20] with some modifications. Silver nitrate solution $(0.01 \mathrm{M})$ was prepared by dissolving $0.16 \mathrm{gm}$ in $100 \mathrm{~mL}$ de-ionized water (solution A). A solution of $0.001 \mathrm{M}$ sodium borohydride was also prepared by dissolving $0.3783 \mathrm{~g}$ solid $\mathrm{NaBH}_{4}$ in $100 \mathrm{~mL}$ distilled water (solution B). 5 $\mathrm{mL}$ of solution $\mathrm{A}\left(0.01 \mathrm{M} \mathrm{AgNO}_{3}\right)$ was added dropwise (1 drop per sec.) to $50 \mathrm{~mL}$ of $0.001 \mathrm{M} \mathrm{NaBH}_{4}$ (solution B) in $250 \mathrm{~mL}$ beaker with drastic stirring at $400 \mathrm{rpm}$ for $30 \mathrm{~min}$ in dark condition. The color change was noted. The biosynthesis of silver NPs was conducted by using Enterobacter cloaca according to Shahverdi et al. [21] with some modifications. Sterilizing nutrient broth was inoculated with Enterobacter cloaca fresh culture (Enterobacter cloaca isolate was isolated from urine of catheterized patients and had the ability to produce biofilm) and incubated in shaker incubator at $150 \mathrm{rpm}, 37^{\circ} \mathrm{C}$ for $24 \mathrm{~h}$. After the incubation period, the broth culture was centrifuged at $6000 \mathrm{rpm}$ for 15 min. in cold centrifuge $\left(4{ }^{\circ} \mathrm{C}\right)$. Supernatant was drawn by pasture pipet and filtrated by Millipore filter 0.4, after which $0.25 \mathrm{~mL}$ of $0.1 \mathrm{M} \mathrm{AgNO}_{3}$ was added to $50 \mathrm{~mL}$ liquid of $10 \mathrm{~mL}$ bacterial supernatant in $40 \mathrm{~mL}$ deionized water. The mixture was incubated in shaker incubator at $150 \mathrm{rpm}, 37^{\circ} \mathrm{C}$ for $24 \mathrm{~h}$. A formed brown color referred to the formation of silver NPs.

\section{Collection of specimens}

The specimens were collected and isolated according to the procedure described in the reference. 28 biofilm bacterial isolates were isolated from urine samples collected from catheterized patients from 4 Iraqi hospitals (Diwaniya, Hilla, Al Qasim, and Al Hashimiya Teaching Hospitals) during the period from February to April 2017 in sterile tubes of $10 \mathrm{~mL}$ and then transferred to laboratory immediately [22].

\section{Bacterial isolate}

S. fonticola and Pantoea sp. were isolated from urine of catheterized patients, which were identified and diagnosed by the automated system VITEK 2 (BioMerieux, Marcy I'Etoile, France) to achieve final diagnostics and identification of the species level. Serratia marcescens and E. coli were diagnosed by biochemical tests as reference strains to Serratia fonticola and Pantoea sp., respectively [22]. The biofilm forming bacteria were investigated through using Congo red agar method [10], tissue culture plate method, and the biofilm forming bacterial was also diagnosed genetically by polymerase chain reaction (PCR) method. Briefly, PCR technique was performed for biofilm formation genes, i.e. smaI and esaI genes in Serratia sp. and Pantoea sp., respectively. The primers were designed by using the National Center for Biotechnology Information (NCBI) gene sequence data base and primer 3 plus design. This primer was provided by Bioneer, South Korea as shown in Electronic Supplementary Information (ESI 1). DNA extraction was carried out according to manufactured instructions of commercial DNA extraction kit (Presto Mini-DNA Bacteria Kit. Geneaid Biotech Ltd. USA). Then, the extracted DNA was estimated by the Nanodrop device at 260/280 nm. PCR master mix was prepared from Accu-Power ${ }^{\circledR}$ PCR-PreMix-Kit master mix reagent according to the company directions (ESI 2). The PCR mixture revealed in ESI 2 was placed in AccuPower PCR-PreMix that contained all PCR components (Taq DNA polymerase, dNTPs, and 10 PCR buffers). Then, all the PCR tubes were transferred into vortex vibration for $3 \mathrm{~min}$ and transferred into thermocycler apparatus (MyGene, Bioneer, Korea). The conditions of the PCR thermocycler are tabulated in ESI 3. Then the products of PCR were analyzed by electrophoresis on a $1 \%$ agarose gel stained with ethidium bromide and observed under a ultra-violet (UV) trans-illuminator.

\section{Antimicrobial susceptibility testing}

The test for antimicrobial susceptibility was achieved by two methods. The first was disc diffusion method, according to Clinical and Laboratory Science Institute (CLSI), 2015 [12]. The test was carried out on Müller-Hinton agar with antibiotics disc listed in Table 5. The second was minimum inhibitory concentration (MIC) testing, accomplished by using VITEK 2 AST system for antibiotics including ampicillin/clavulanic, 
cefazolin, ceftazidime, ceftriaxone, cefepime, ertapenem, imipenem, gentamicin, tobramycin, ciprofloxacin, levofloxacin, nitrofurantoin, and trimethoprim/sulfamethoxazole. The results in both methods were regarded as sensitive, intermediate or resistant based on the Clinical and Laboratory Science Institute (CLSI) standard guidelines, M100, 2017 [13].

\section{Statistical analysis}

All experiments were carried out in triplicate to validate the reproducibility of experiments. Statistical analysis was conducted by using one way ANOVA at p-value 0.05 by SPSS Statistics 24.0 software.

\section{Results and Discussion Synthesis and characterization of silver nanoparticles}

Silver NPs were prepared according to reduction of silver nitrate with sodium borohydride as a reducing agent and capping agent. A brown colour was formed, representing the formation of silver NPs as shown in Fig. 1(a), and the colour was also developed to be dark brown when continuing mixing with magnetic stirrer for $0.5 \mathrm{~h}$. Additionally, biosynthesized silver NPs were also prepared through incubation of Enterobacter cell free filtrate solution (pale yellow) with silver nitrate
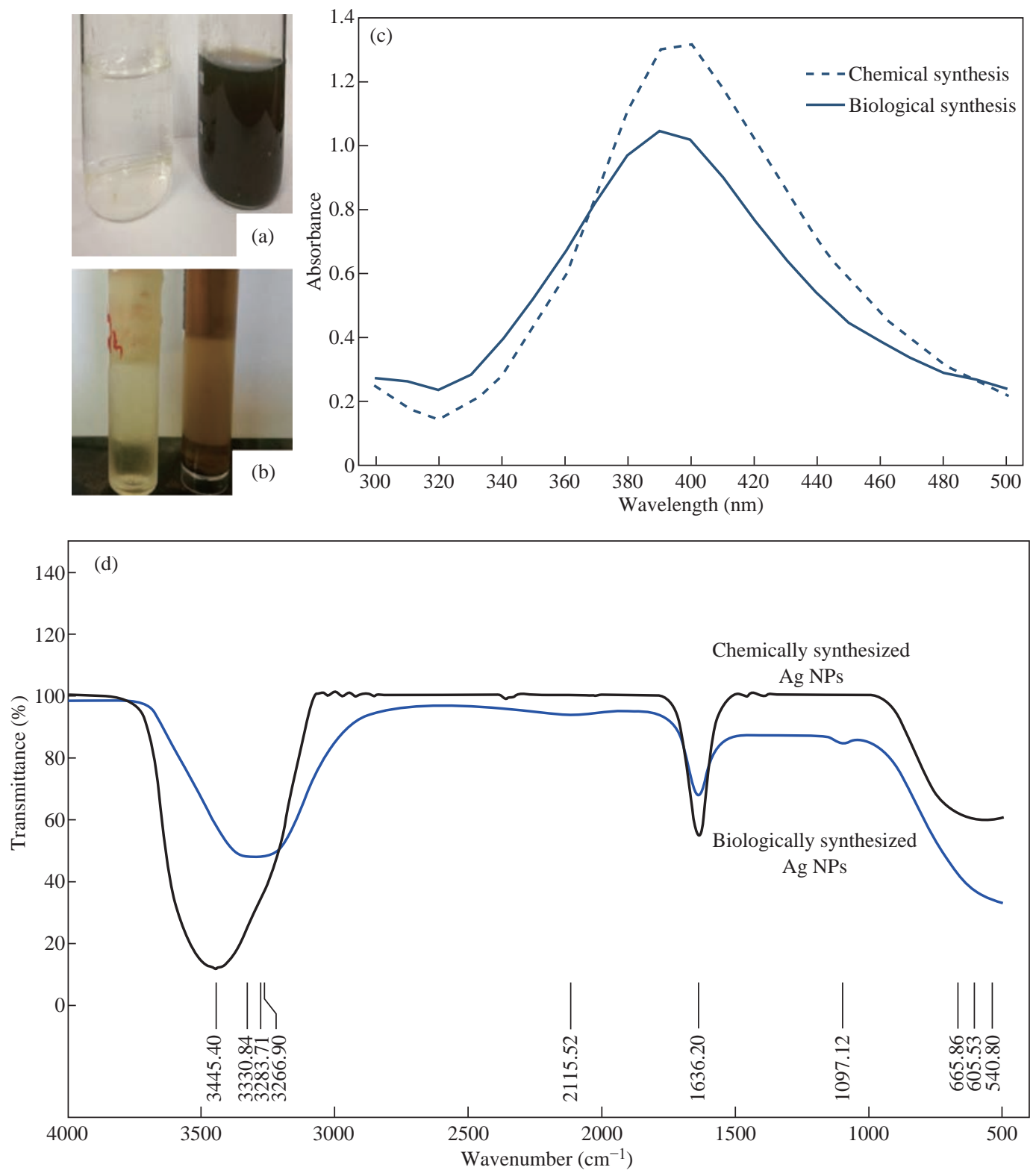

Fig. 1 (a) Colour profile for the synthesis of silver NPs using chemical method through reduction of silver ions with sodium borohydride as a reducing agent. (b) Colour profile for the biosynthesis of silver NPs using secondary metabolites of bacteria. (c) UVvisible absorption spectrum of chemically and biologically synthesized silver NPs. And (d) FTIR spectrum of silver NPs which were synthesized with chemical and biological methods. 
solution for $24 \mathrm{~h}$, leading to forming brown colour as stated in Fig. 1(b). The change in colour was owing to the reduction of $\mathrm{Ag}^{+}$ions to Ag NPs. The colour change was previously reported in extracellular synthesis method from bacterial source [23] and from Enterobacter cloacae specially [21]. The brown colour for both methods related to excitation of SNPs surface Plasmon vibration [24], as a result of SNPs production.

Fig. 1(c) represents the absorption spectrum of both chemical and biological synthesized silver NPs. In chemical SNPs, the UV-visible absorption spectra revealed the absorption band was at $390 \mathrm{~nm}$ due to surface Plasmon resonance of silver NPs. The absorption energy of SNPs depended on Plasmon resonance degree which represented the ratio of silver ion to silver zero valent [25]. The observation of biological SNPs synthesis by $E$. cloacae was strong with narrow peak at wavelength $400 \mathrm{~nm}$. The peak indicated to narrow size range of NPs which was less than $100 \mathrm{~nm}$ [21]. The surface Plasmon attributed the vibration combination of silver NPs free electrons with light wave, and it was considered as another indicator of NPs production at the range of absorption from 390-420 nm [26]. The synthesized silver NPs were also characterized by using Fourier transform infrared spectroscopy (FTIR) as seen in Fig. 1(d). The FTIR results illustrated FTIR spectrum of chemical and biological synthesized SNPs respectively in the wavelength range from 500 to $4000 \mathrm{~cm}^{-1}$. It can be shown in the figure that the FTIR spectrum presented absorption bands at 1635, 1636 and 3330, $3445 \mathrm{~cm}^{-1}$ in chemical and biological synthesis, respectively. The band at 1636 and1635 $\mathrm{cm}^{-1}$ referred to vibration stretching group $\mathrm{C}=\mathrm{O}$ indicating the carboxylic acids group which was bounded to silver NPs. The peak at 3445 and $3330 \mathrm{~cm}^{-1}$ belonged to O-H stretching vibration band for polyphenolic compounds in the extract adsorbed on the surface of biological synthesized NPs, as well as the presence of water into the system, and thereby intermolecular hydrogen-bonding in case of chemically synthesized silver NPs. All these stretching vibrations bands clarified that carboxylic groups and polyphenolic groups were coated on the surface of silver NPs producing stabilized NPs with negligible aggregation [27].

Silver NPs size of both methods were determined by dynamic light scattering. The size of NPs distribution analysis of chemical method revealed the average of particles size was approximately 22-28 nm (25 \pm 3 ) with zeta potential of about -48 (Fig. 2(a) and (b)), while the biosynthesis SNPs was 56-70nm $(63 \pm 7)$ with zeta potential of -30, as seen in Fig. 2(c) and (d). Concerning the antibacterial activity of SNPs influence of its size particles, the small particles are well known to be more effective than larger ones in terms of antibacterial and anti-biofilm activities [28]. Many previous studies reported that antibacterial activity was based on particles size of SNPs [29]. Fig. 2(e) and (f) revealed typical SEM micrograph of chemical and biological silver NPs obtained by the reduction of $\mathrm{AgNO}_{3}$ solution with sodium borohydride and cell-free filtrate of E. cloacae, respectively. The morphology of NPs was cubic in shape, uniformly (mono dispersed) without significant aggregation in both types. The particle size ranged from 22-28 nm for chemical silver NPs and 56-72 nm for biological SNPs. Scanning electron microscopy (SEM) was employed to determine the shape, size and morphology of the chemically and biologically synthesized silver NPs.

\section{Antimicrobial effects of silver NPs on biofilm bacteria}

Antibacterial activity of silver NPs were evaluated by many previous studies [30, 31], but fewer studies focused on the anti-biofilm activity of SNPs such as $[32,33]$, and the comparative effect of both types (chemical and biological SNPs). Silver NPs were recorded to be anti-biofilm agents which were effective against biofilm bacteria formation, and were also most effective against multi- drug resistant bacteria, resulting from biofilm formation to solve most serious problem to worldwide public health [28, 34, 35]. Herein, the antimicrobial activity of chemo and biosynthesized SNPs on S. fonticola and Pantoea sp. was evaluated using well diffusion agar and MIC methods as shown in Fig. 3. The antimicrobial effects of both types SNPs on S. fonticola and Serratia sp. as a control are shown in Fig. 3(a) and (b). The antimicrobial effect of both types SNPs on Pantoea sp. and E. coli as control were illustrated in Fig. 3(c) and (d).

The results in Fig. 3 revealed that the antibacterial activity of SNPs increased directly with the concentration increasing for both types. The inhibition zone diameter of tested biofilm bacteria increased significantly with the increasing of concentration of SNPs. Tiwari et al. pointed out that protein leakage from bacterial cell treated with SNPs increased along with SNPs concentration, which led to cell death, and the releasing of protein was lower in gram positive than negative bacteria [36]. Gram negative biofilm bacteria (S. fonticola and Pantoea sp.) were more susceptible 

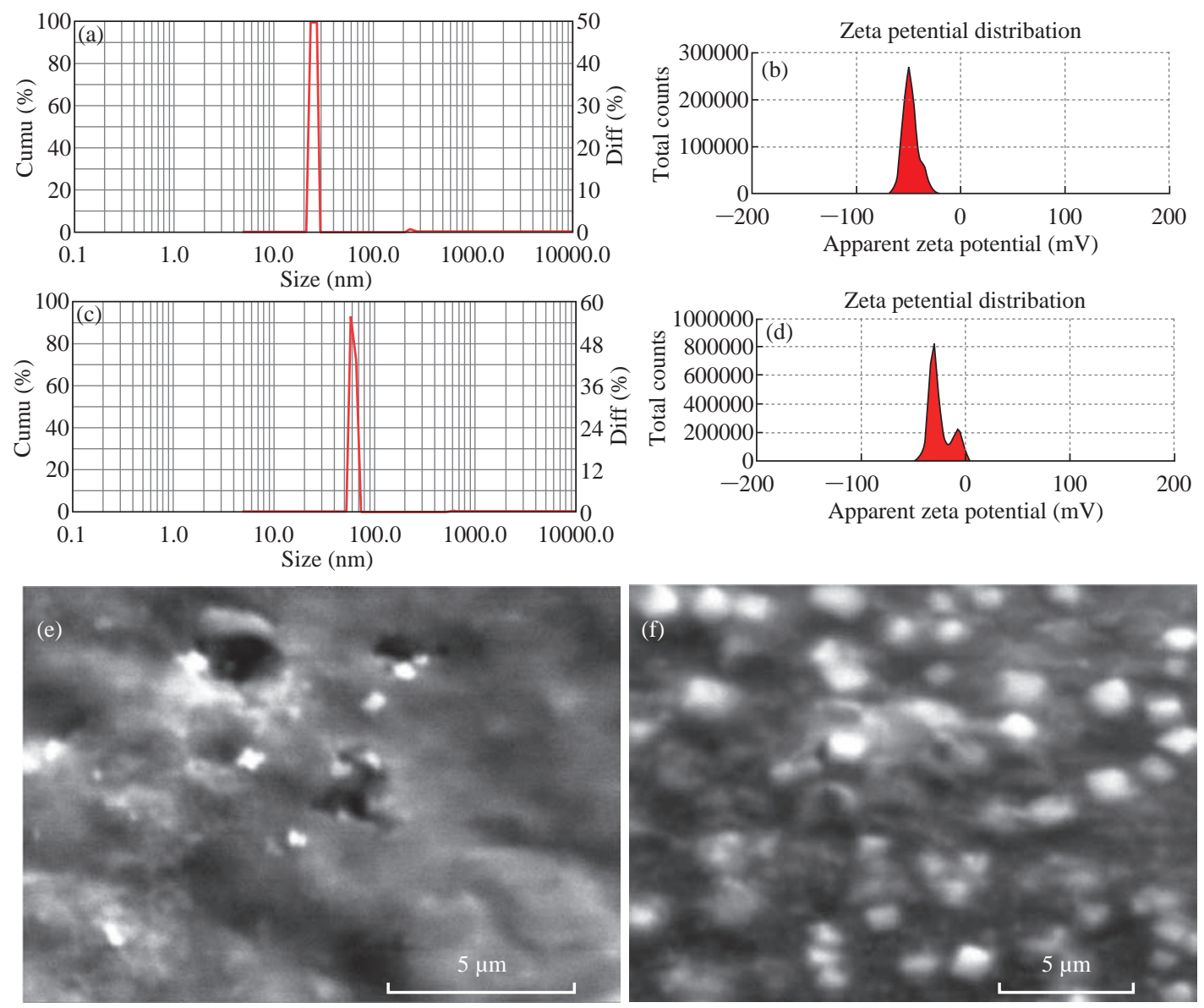

Fig. 2 (a), (b) The average particle diameter and zeta potential of chemosynthesis of silver NPs. (c), (d) The average particle diameter and zeta potential of biosynthesis of silver NPs. (e), (f) Scanning electron microscopy (SEM) of chemically and biologically synthesized silver NPs, respectively explaining the crystallized particles of nanosized silver formed.
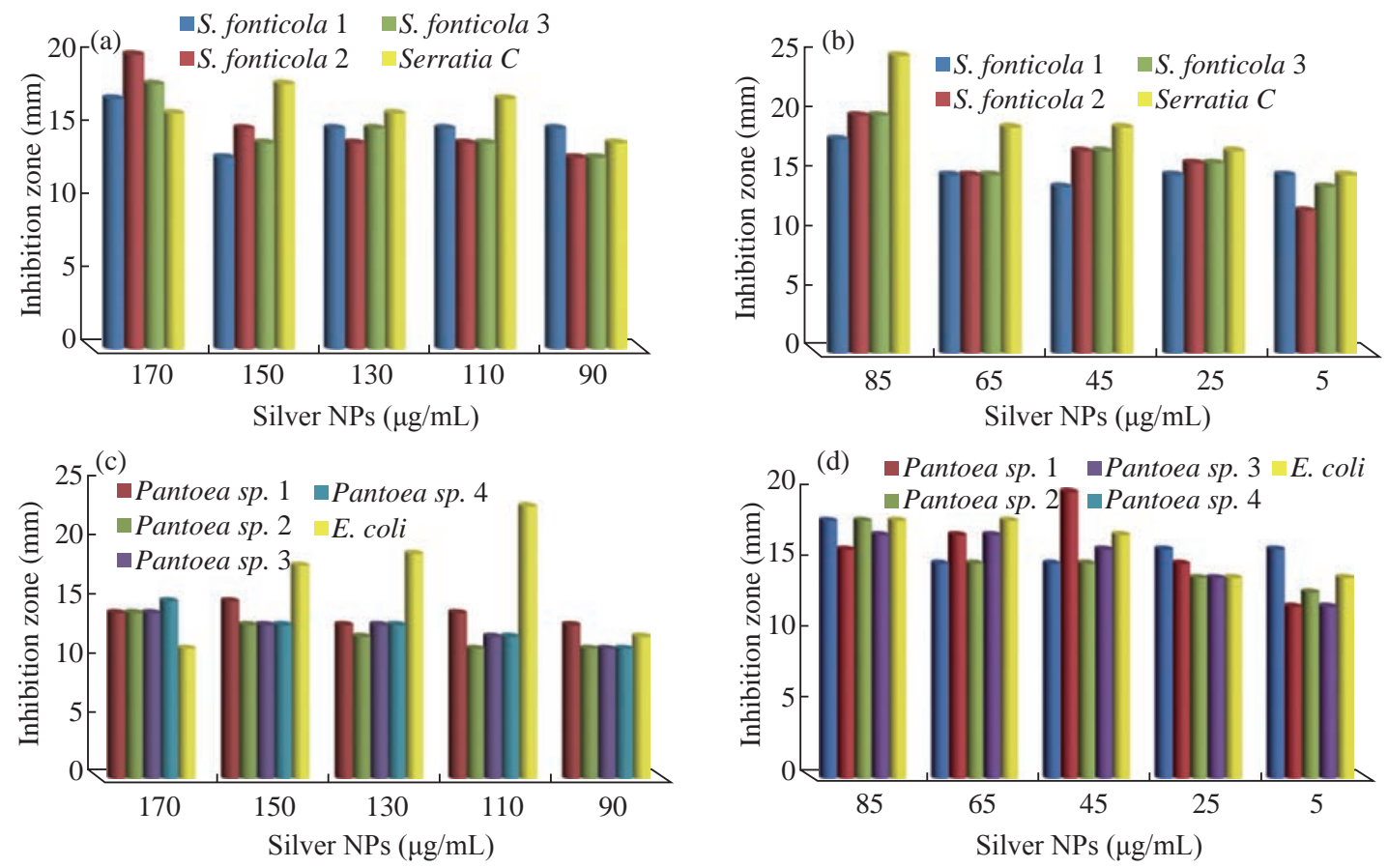

Fig. 3 (a), (c) Antibacterial activity of different concentrations of chemically synthesized silver NPs expressed as inhibition zone. (b), (d) Antibacterial activity of different concentrations of biosynthesized silver NPs expressed as inhibition zone. The chemosynthesis of silver NPs was prepared by adding sodium borohydride to silver nitrate, while the biosynthesis of silver NPs was conducted through addition of biomass free filtrate into silver nitrate, which stayed overnight to produce a brown colour. 
to SNPs in both types. The figure also shows that the MIC values of biological silver NPs towards both $S$. fonticola and Pantoea sp. isolates were $25 \mu \mathrm{g} / \mathrm{mL}$, while for chemical silver NPs were $90 \mu \mathrm{g} / \mathrm{mL}$ to all isolates. Solutions of these values were used later in RT-PCR analysis to evaluate the gene expression among tested bacteria. It was supposed that SNPs caused pits in gram negative cell wall leading to increasing permeability of cell membrane and inhibiting respiratory chain leading to kill treated bacterial $[37,38]$. The antimicrobial effect of silver NPs may be related to attachment to the surface of cell membrane disturbing permeability and respiration functions of the cell [39]. It is also possible that silver NPs not only interact with the surface of membrane, but can also penetrate inside the bacteria [40]. Many researchers also proposed that Ag+ ions interacted with the thiol groups in bacteria proteins, affecting the replication of DNA [41]. It has been reported that Ag+ ions uncouple the respiratory chain from oxidative phosphorylation or collapse the protonmotive force across the cytoplasmic membrane [42]. Table 1 depicts the statistical analysis for antimicrobial effect of chemical SNPs, which illustrates that there was no significant differences among $S$. fonticola isolates ( $p=0.229)$ against control as well as Pantoea sp. isolates $(p=0.171)$ and its control. On the other hand, biologically synthesized silver NPS also showed no significant differences $p=(0.254,0.959)$ in biological SNPs, respectively.

\section{Synergistic effect of silver NPs combined with imipenem on biofilm isolates}

The antibacterial activity of silver NPs was enhanced through combination with some antibiotics. Herein, imipenem antibiotic was utilised in combination with both chemical and biological silver NPs. Fig. 4 shows

Table 1 Statistical characteristics analysis of antibacterial activity of chemosynthesis and biosynthesis of silver NPs with reference to zone of inhibition for both bacteria isolates S. fonticola and Pantoea sp.

\begin{tabular}{cccc}
\hline & & Bacteria IZ \pm SE & Control IZ \pm SE \\
\cline { 2 - 4 } Biological silver NPs & S. fonticola & $15.933 \pm 0.564$ & $17.400 \pm 1.029$ \\
& Pantoea sp. & $15.550 \pm 0.450$ & $15.600 \pm 0.678$ \\
Chemical silver NPs & S. fonticola & $15.00 \pm 0.507$ & $16.200 \pm 0.663$ \\
& Pantoea sp. & $12.850 \pm 0.283$ & 0.959 \\
\hline
\end{tabular}

Note: IZ = inhibition zone; $\mathrm{SE}=$ standard error

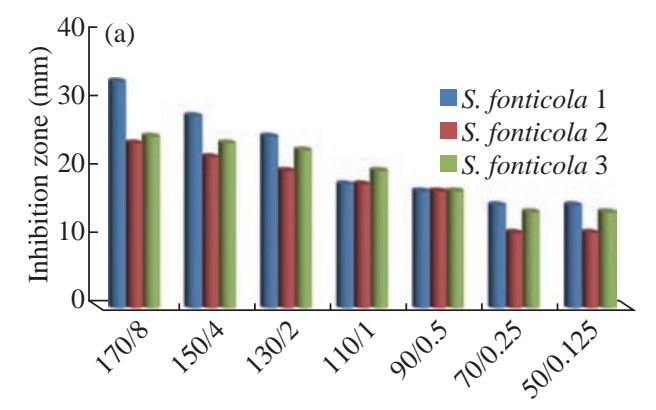

Silver NPs/Imipenem $(\mu \mathrm{g} / \mathrm{mL})$

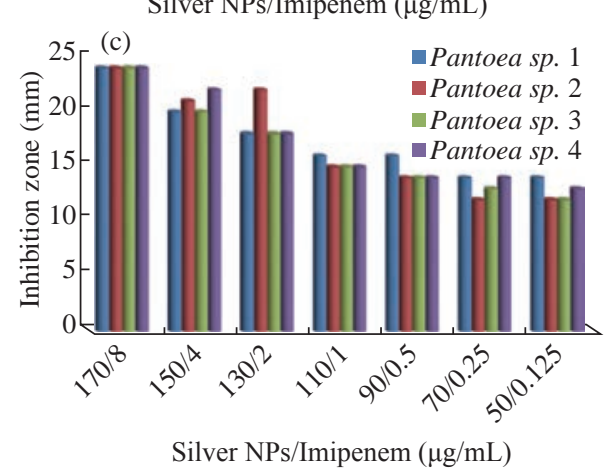

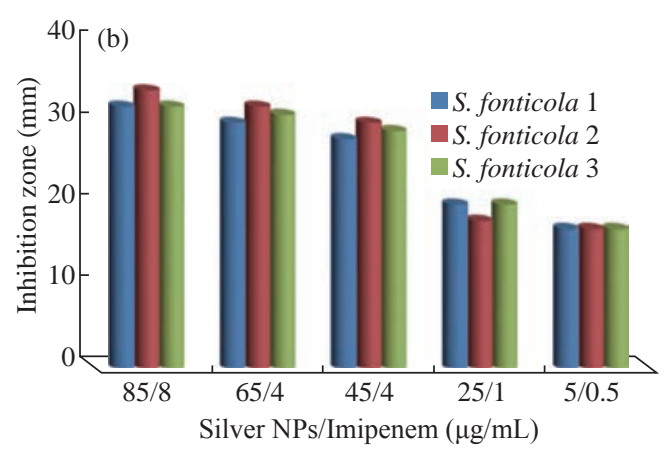

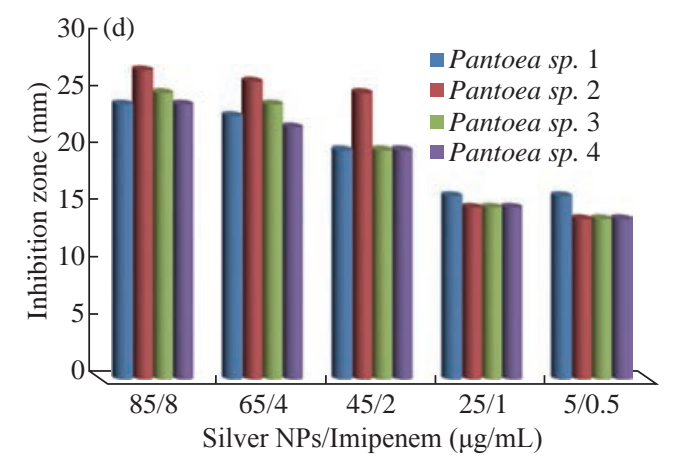

Fig. 4 (a), (c) Antibacterial activity of different concentrations of chemically synthesized silver NPs combined with varied concentrations of imipenem antibiotic expressed as inhibition zone. (b), (d) Antibacterial activity of different concentration of biosynthesized silver NPs combined with varied concentrations of imipenem antibiotic expressed as inhibition zone. The chemosynthesis of silver NPs was prepared by adding sodium borohydride to silver nitrate, while the biosynthesis of silver NPs was conducted through addition of biomass free filtrate into silver nitrate, which stayed overnight to produce a brown colour. 
the antibacterial activity of chemically and biologically synthesized silver NPs. It can be seen in the figure that the antibacterial action profoundly increased for both categories of silver NPs and enhanced the growth inhibition of tested biofilm bacteria. Thus, the antibacterial effect of combined silver NPs and antibiotic exhibited higher than bare SNPs or free antibiotic. These results corresponded to references [26] and [43] where those researchers pointed out that the combined antibiotics with SNPs caused greater anti-biofilm activity and elevated bacterial cell death level. Therefore, the treatment with combination of antibiotics and SNPs could be considered of more potent effectiveness as antibacterial and anti-biofilm. Namasivayam et al. also concluded that silver NPs made a good compatibility in combination with antibiotics to inhibit bacterial biofilm [44]. Therefore, silver NPs can be used as great adjuvants to antibiotics upon combination by enhancing antibiotics activity against gram positive and negative bacteria [43]. It has been recently reported that the combination of silver NPs and vancomycin increased the inhibition of biofilm activity to $55 \%$ and $75 \%$ to gram positive and negative bacteria, respectively [26]. Table 2 depicts the statistical analysis of results of the combination of both types of silver NPs with imipenem. These results have shown that biological silver NPs in combination with imipenem were more effective than chemical silver NPs with imipenem, with significant differences at $p=0.005$ and 0.028 for S. fonticola and Pantoea sp., respectively.

The results of individual combination of both types of silver NPs with imipenem, revealed that there was a synergistic effect. Table 3 shows a highly synergistic effect and anti- biofilm activity mostly more than each one alone and to both types (chemical and biological) and to all tested biofilm bacteria. Anti-biofilm activity of silver NPs in other studies increased to reach 50\% and $70 \%$ to gram positive and negative biofilm forming bacteria, respectively, when combined with ampicillin [26]. In addition, it increased bacterial susceptibility to antibiotics when combined with them as synergistic effect especially in biofilm infection like nitrofurazone increased its effect in the presence of silver [45].

\section{Gene expression of biofilm formation incubated with silver NPs}

Biofilm formation is a cooperative genetic process requiring different genes to cause and regulate biofilm formation. NPs may potentially effect on the process, and so the goal was to evaluate the silver NPs' effect on gene expression level of biofilm causative and regulatory

Table 2 Statistical characteristics analysis of antibacterial activity of combination of chemosynthesized and biosynthesized silver NPs individually with imipenem antibiotic with reference to zone of inhibition for both bacteria isolates of $S$. fonticola and Pantoea sp.

\begin{tabular}{ccc}
\hline Bacteria & Chemical SNPs + imipenem $(\mathrm{IZ} \pm \mathrm{SE})$ & Biological SNPs + imipenem(IZ \pm SE) \\
\hline S. fonticola & $19.571 \pm 1.237$ & $25.800 \pm 1.718$ \\
Pantoea sp. & $17.071 \pm 0.778$ & $19.950 \pm 1.03$ \\
\hline
\end{tabular}

Note: $\mathrm{IZ}=$ inhibition zone; $\mathrm{SE}=$ stander error

Table 3 Synergistic effect of combination of different concentration of both chemically synthesized and biologically synthesized silver NPs with different concentration of imipenem antibiotic towards bacterial isolates S. fonticola and Pantoea sp.

\begin{tabular}{|c|c|c|c|c|c|c|c|c|}
\hline \multirow{5}{*}{ 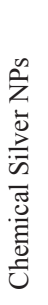 } & Conc. & S. fontic 1 & S. fonti 2 & S. fonti 3 & Pantoea 1 & Pantoea 2 & Pantoea 3 & Pantoea 4 \\
\hline & $170 / 8$ & 94 & 20 & 38.8 & 71.4 & 71.4 & 71.4 & 60 \\
\hline & $150 / 4$ & 115 & 46 & 71.4 & 33.3 & 61.5 & 53.8 & 69.2 \\
\hline & $130 / 2$ & 66.6 & 42 & 53.3 & 28.5 & 83.3 & 38.4 & 38.4 \\
\hline & $110 / 1$ & 20 & 38 & 28.5 & 14.2 & 36.3 & 7.1 & 25 \\
\hline \multirow{6}{*}{ 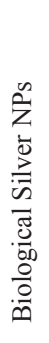 } & $90 / 0.5$ & 13 & 30.7 & 30.7 & 23 & 27.2 & 27.2 & 27.2 \\
\hline & $85 / 8$ & 77.7 & 70 & 60 & 33.3 & 68.7 & 38.8 & 41.1 \\
\hline & $65 / 4$ & 100 & 113 & 106 & 53.3 & 52.9 & 60 & 29.4 \\
\hline & $45 / 2$ & 100 & 76.4 & 93.3 & 33.3 & 25 & 33.3 & 25 \\
\hline & $25 / 1$ & 33.3 & 12.5 & 17.6 & 0 & 0 & 7.1 & 7.1 \\
\hline & $5 / 0.5$ & 13.3 & 41.6 & 21.4 & 0 & 16.6 & 7.1 & 16 \\
\hline
\end{tabular}


genes. Depending on previous literature about the gene expression of biofilm for the bacterial isolates under study, no study was found to be dealing with this subject except one that used chitosan to inhibit staphylococci biofilm formation by down-regulating icaA gene expression [46]. Hence, the current study could be considered the first one that applied the SNPs prepared chemically and biologically to minimize the biofilm formation in bacterial infection caused on catheterized patients. For this reason, the discussion was limited to interpretation of the recent results. The results of total RNA were estimated by Nanodrop spectrophotometer as shown in Table 3-15 (S. fonticola and Pantoea sp.), which explained the total RNA concentration ranged from 384.6 to $634.3 \mathrm{ng} / \mu \mathrm{L}$, and the RNA purity was at the ratio of $260 / 280 \mathrm{~nm}$ ranging in 1.71-1.89. These data emphasized that the technique used for RNA extraction was perfect for amplification in the RTPCR system. Total RNA was extracted by using Total RNA extraction Trizol kit in performing RT-qPCR for relative gene expression analysis of biofilm genes smaI and esaL for the tested and the control of S. fonticola and Pantoea sp., respectively in different treatments. The concentration and purity of the extracted RNA had powerful influence on gene expression outcome and determined the accuracy of RNA profile. RNAs are characterized by sensitive molecules as compared to DNAs, which can be easily denaturized by heating, UV or nuclease, and so RNA sample should be free of protein, nucleases and inhibitor enzyme or contaminated with DNA molecules [47]. Combination of the reverse transcription with PCR to be RT-PCR made a powerful gene expression quantification method [48]. To gain an insight into molecular estimation of differences in gene expression of biofilm regulation by smaI and esaL genes in the test and the control of S. fonticola and Pantoea sp. isolates, respectively, quantitative reverse transcription real-time PCR (RTqPCR) was performed. The gene expression patterns were evaluated in biofilm bacterial genes before and after treatment with antibiotics and silver NPs alone and combined, by normalization with expressions of the housekeeping gene (reference gene), RNA polymerase $\beta$ subunit gene (rpoB). Reference gene was potent to assess the accuracy of tested gene [49] and must be expressed constantly according to experimental conditions [48]. RT-PCR was considered a standard analysis technique to assess gene expression of potential gene which impacted on pathological diseases [50]. The current study investigated the expression stability of rpob housekeeping genes which highly conserved RNA subunit as reference genes to study the gene expression of biofilm regulated and causative genes (smaI and esaL) in S. fonticola and Pantoea sp., and to determine the differences in gene expression of biofilm gene before and after treatment with silver NPs and antibiotics.

The relative quantification meant the change of target gene expression relative to the control group [48]. Quantification PCR estimates the amplification of sampling DNA at cycle number according to the induction of fluorescence indicator (SYBR Green), which was used as amplification detection of target genes and assessment of expression levels [50]. Fig. 5(a)-(d) show the RT-qPCR amplification biofilm genes of the tested bacteria and the control. The results described in the figure revealed that gene expression of biofilm genes (smaI and esaL) could be underexpressed when biofilm formation bacterial cell ( $S$. fonticola and Pantoea sp.) was treated with chemical and biological silver NPs, antibiotics (imipenem), and the combination of them which might lead to loss of its biofilm ability. The expression was termed "RFU", meaning "relative fluorescence units", is a measurement unit used in RT-PCR analysis to detect fluorescence signal [47]. The cycle number across red line is called the threshold cycle, or $C_{\mathrm{T}}$, which represents the cycle number of PCR that gene expression occurs [51]. $\mathrm{C}_{\mathrm{T}}$ is a value of PCR cycle at which the fluorescent signal determines the amplification of each gene by monitoring it to represent expression level [50].

The relative quantification gene expression was determined according to $C_{\mathrm{T}}$ value of the tested and the housekeeping gene and differences between them, to determine the fold change in gene expression [50] based on the $\triangle \mathrm{CT}$ method reference gene equation which is simpler to perform than the Livak method and gives the same results [51]:

Ratio (reference/target) $=2^{\wedge} C_{\mathrm{T}}$ (reference) $-C_{\mathrm{T}}$ (target) $=$ fold change.

Fold changes were represented by relative gene expression of target genes (icaA, smaI and esaL) that normalized to reference gene rpoB. The reference gene utilised to normalize the target gene expression was very important to estimate the fold change in gene expression and the accurate results, because is considered a highly conserved expressed RNA subunit and used as constant standard [52]. Calculations of the fold change gene expression of differences between the tested $C_{\mathrm{T}}$ and the housekeeping gene are shown in Fig. 

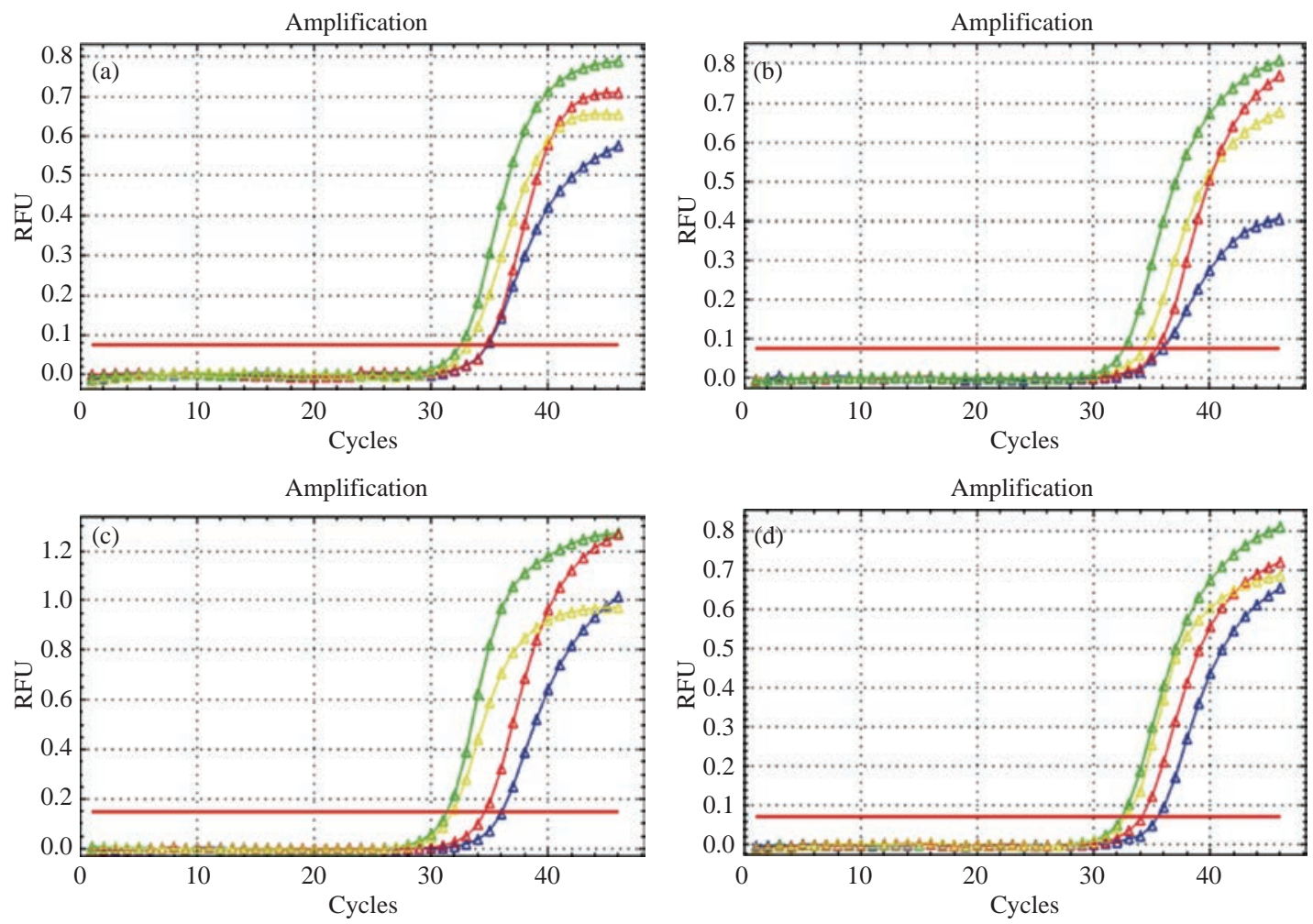

Fig. 5 RT-qPCR amplification biofilm formation genes of (a) smaI gene in treated and untreated S. fonticola 1, 2, (b) smaI gene in treated and untreated S. fonticola 3, (c) esaL gene in treated and untreated Pantoea sp. 1, 2, and (d) esaL gene in treated and untreated Pantoea sp. 3, 4. Red plot $=\mathrm{T} 1$ (chemical silver NPs), blue plot $=\mathrm{T} 2$ (biological silver NPs), yellow plot $=$ T3 (imipenem), green plot $=$ control, and red line $=$ threshold line.
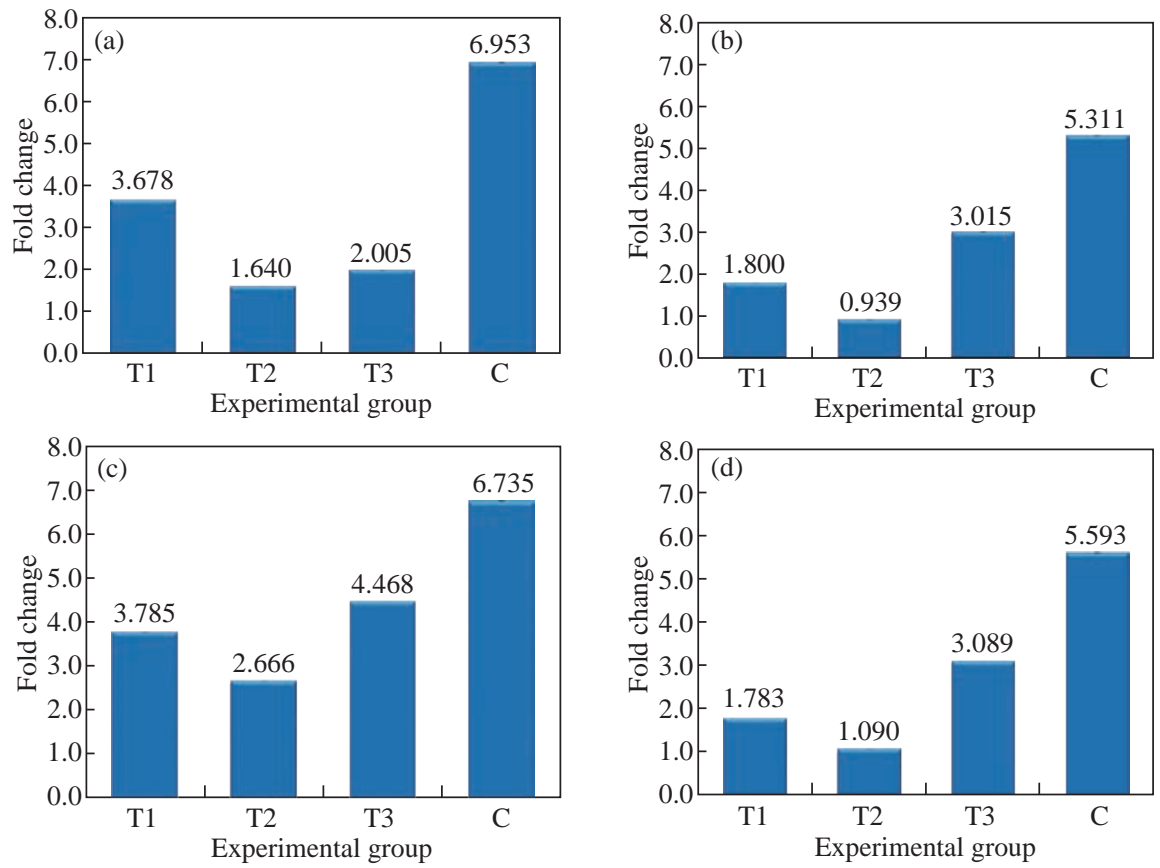

Fig. 6 Mean fold change of gene expression of (a) smaI in biofilm of S. fonticola 1, 2, (b) smaI in biofilm of S. fonticola 3, (c) esaL in biofilm of Pantoea sp. 1, 2, and (d) esaL in biofilm of Pantoea sp. 3, 4. T1 = chemical silver NPs, T2 = biological silver NPs, T3= imipenem, and $\mathrm{C}=\operatorname{control}(S$. fonticola with broth + sugar $)$.

6(a)-(d), which represent the expression level of smaI and esal during biofilm formation. The level of smaI and esal expression decreased dramatically for S. fonticola and Pantoea sp. Isolates, respectively incubated with chemical and biological silver NPs and selected antibiotics (imipenem) (with varying influences) in comparison to the level of gene expression of the control isolates (broth of biofilm bacteria with sugar 
and without silver NPs or antibiotics treating). The fold change of smaI expression level in S. fonticola isolate 1, 2 ranged from 1.640 to 3.678 as compared to 6.953 for the control, while it ranged from 0.939 to 3.015 against the control in S. fonticola isolate 3, as shown in Fig. 6 (a) and (b). Also, the fold change of esaL expression in Pantoea sp. isolates 1, 2 ranged from 2.666 to 4.468 versus 6.735 for the control, and for Pantoea sp. isolates 3, 4, it ranged from 1.090 to 3.089 in comparison to 5.593 for the control. These results might explain that silver NPs in both types and antibiotics mechanisms down-regulated gene expression of smaI and esaL genes, and might degrade the quarm sensing signal which regulated biofilm formation.

Fig. 6 (a)-(d) also show that biological silver NPs were more influential on gene expression of the tested biofilm bacterial genes than chemical silver NPs or antibiotics, with the significant difference $p=0.009$, 0.001, 0.006 and 0.007 to biofilm bacterial isolates (S. fonticola 1, 2, S. fonticola 3, Pantoea sp. 1, 2, and Pantoea sp. 3, 4), respectively (Appendix 1-5). Genotypic effects were associated with phenotypic effects of biological silver NPs on the tested biofilm bacteria, which revealed more potent antibacterial effects than the others.

\section{Gene expression of biofilm formation for antibiotic combined silver NPs}

At genetic level, as well as at phenotypic level, the gene expression was influenced with the combination which down-regulated the expression of the tested genes compared to individual antimicrobial agents, as shown in Fig. 7 for the combination of chemical silver NPs with antibiotic imipenem and Fig. 8 for the combination of biological silver NPS with antibiotic imipenem. The results revealed that gene expression of biofilm encoding genes (smaI and esaL) decreased due to the combining effect of chemical and biological silver NPs with imipenem. Each treatment was a microdilution broth of mixing silver NPs with antibiotic at below, equal and above MIC concentrations.

Combined therapies potentially has many benefits compared to individual antibiotic therapies, especially in the cases of severe infections. Combinations would increase the effectiveness of antimicrobial agents through synergism, increase the range of bacteria targeted in empirical therapy through affecting different targets, limit virulence factors expression, and prevent the development of antibiotic resistance [53]. Fig. 7(a) and (b) depict that the smaI expression level decreased from 7.4 to 0.46 in accordance with excess of the combination of chemical silver NPs and imipenem concentration, compared to the control of S. fonticola bacteria isolates1, 2. The same was true for $S$. fonticola bacteria isolate 3 . The smaI expression level decreased from 9.95 to 0.16 in accordance with excess of the combination of chemical silver NPs and imipenem concentration in comparison to the control, as seen in Fig. 7 (c) and (d). However, for esal gene, the expression level decreased from 4.47 to 1.65, in accordance with excess of the combination of chemical silver NPs and imipenem concentration as compared to the control Pantoea sp. isolates1, 2 (Fig. 7 (e) and (f)). Fig. 7 (g) and (h) show the esaL expression level decreased from 5.13 to 0.58 in accordance with excess of the combination of chemical SNPs and imipenem concentration in compared to the control Pantoea sp. isolates 3, 4. Upon the combination with biosynthesized silver NPs, the smaI expression level decreased from 6.2 to 1.0 in accordance with excess of the combination of biological silver NPs and imipenem concentration in comparison to the control S. fonticola isolates 1, 2 (Fig. 8 (a) and (b)). Also, the smaI expression level decreased from 6.68 to 0.23 in accordance with excess of the combination of biological silver NPs and imipenem concentration in comparison with the control $S$. fonticola isolates 3, 4, as illustrated in Fig. 8 (c) and (d). For Pantoea sp. isolates 1,2 , the esaL expression level decreased from 2.69 to 0.25 in accordance with excess of the combination of biological silver NPs and imipenem concentration in comparison to the control, as stated in Fig. 8 (e) and (f). At last, the esaL expression level decreased from 5.13 to 1.9 in accordance with excess of the combination of biological silver NPs and imipenem concentration in comparison to the control Pantoea sp. isolates 3, 4, as described in Fig. 8 (g) and (h).

Statistical analysis revealed that the combination between chemical and biological silver NPs with imipenem on $S$. fonticola 1,2 isolates exhibited a significant difference $p=0.002$ to chemical SNPs combination only (Appendix 6, 7). But S. fonticola 3 showed a significant differences $p<0.001$ in chemical and biological silver NPs combination (Appendix 8, 9). The combination effect of chemical and biological silver NPs with imipenem on Pantoea sp. isolates revealed a significant difference $p<0.001$ in 

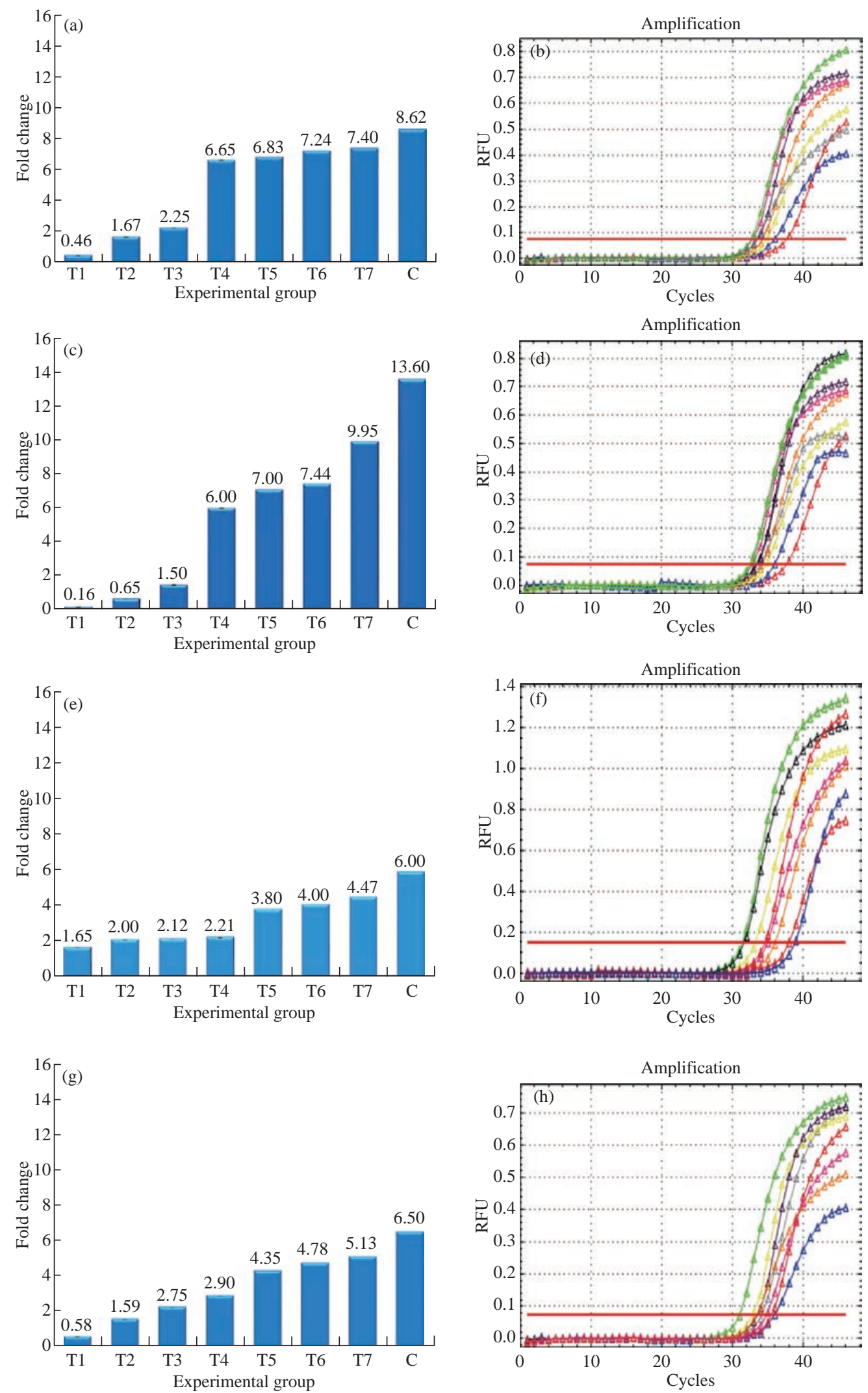

Fig. 7 Mean of fold change in gene expression of (a) smaI in biofilm of S. fonticola 1, 2, (c) smaI in biofilm of S. fonticola 3, (e) esaL in biofilm Pantoea sp. isolates 1, 2, and (g) esaL in biofilm Pantoea sp. isolates 3, 4, separately treated with the combination of chemical SNPs and imipenem. T1 $=170 / 8, \mathrm{~T} 2=150 / 4, \mathrm{~T} 3=130 / 2, \mathrm{~T} 4=110 / 1, \mathrm{~T} 5=90 / 0.5, \mathrm{~T} 6=70 / 0.25, \mathrm{~T} 7=50 / 0.125, \mathrm{C}=$ control (S. fonticola broth and sugar). RT- qPCR amplification biofilm formation genes in treated and untreated (b) smaI in biofilm of $S$. fonticola 1, 2, (d) smaI in biofilm of S. fonticola 3, (f) esaL in biofilm of Pantoea sp. isolates 1, 2, and (h) esaL in biofilm of Pantoea sp. isolates 3, 4. Red plot $=\mathrm{T} 1$, blue plot $=\mathrm{T} 2$, yellow plot $=\mathrm{T} 3$, orange plot $=\mathrm{T} 4$, black plot $=\mathrm{T} 5$, pink plot $=\mathrm{T} 6$, grey $p l o t=\mathrm{T} 7$, green plot $=$ control, and red line $=$ threshold line. 

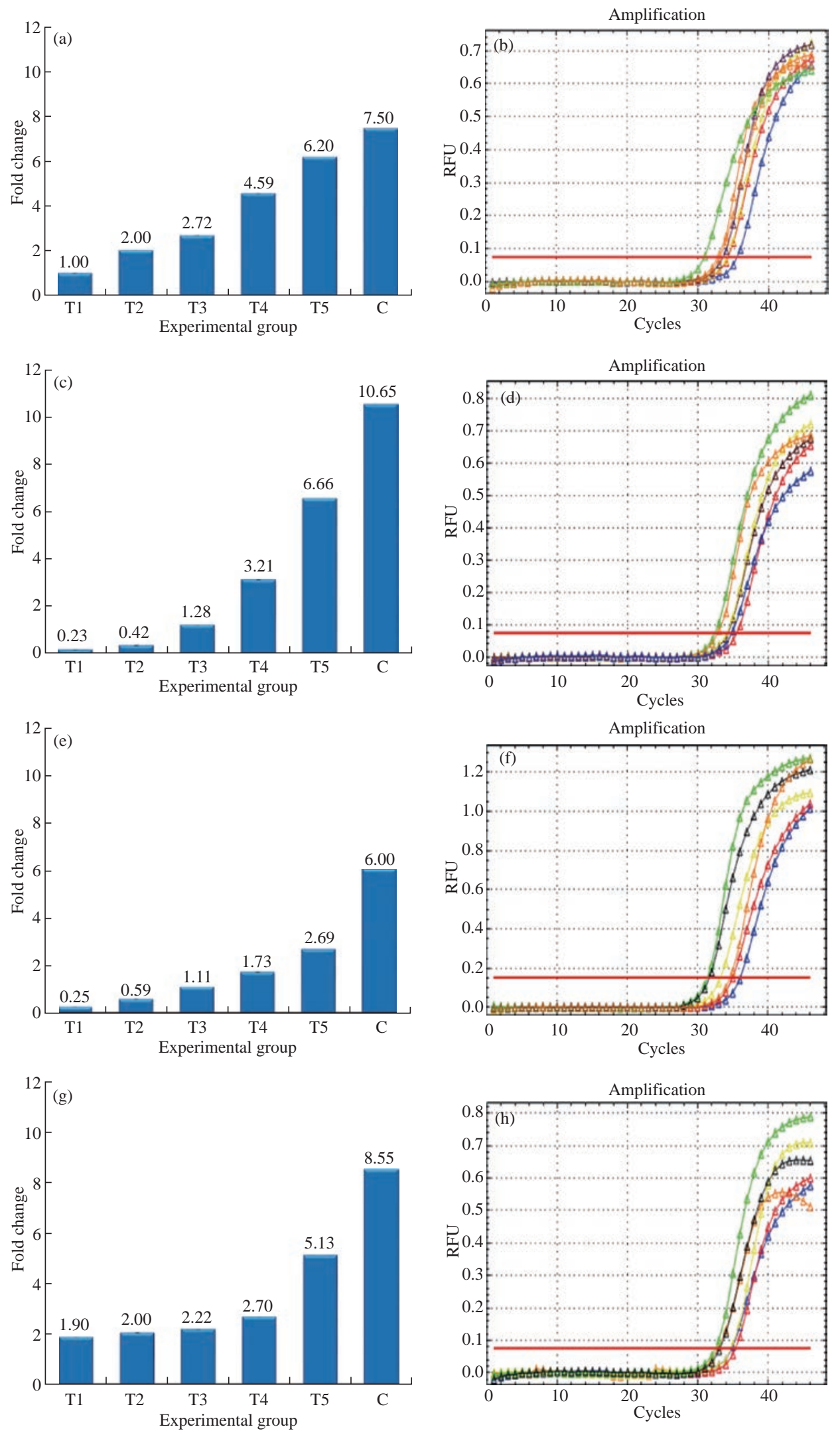

Fig. 8 Mean of fold change in gene expression of (a) smaI in biofilm of S. fonticola 1, 2, (c) smaI in biofilm of S. fonticola 3, (e) esaL in biofilm Pantoea sp. isolates 1, 2, and (g) esaL in biofilm Pantoea sp. isolates 3, 4, separately treated with combination of biosynthesized SNPs and imipenem. T1 = 170/8, T2 =150/4, T3 =130/2, T4 =110/1, T5 = 90/0.5, T6 = 70/0.25, T7 = 50/0.125, C $=$ control $(S$. fonticola broth and sugar). RT- qPCR amplification biofilm formation genes in treated and untreated (b) smaI gene in biofilm of S. fonticola 1, 2, (d) smaI gene in biofilm of S. fonticola 3, (f) esaL gene in biofilm of Pantoea sp. isolates 1, 2, and (h) esaL gene in biofilm of Pantoea sp. isolates 3, 4. Red plot $=\mathrm{T} 1$, blue plot $=\mathrm{T} 2$, yellow plot $=\mathrm{T} 3$, orange plot $=\mathrm{T} 4$, black plot $=\mathrm{T} 5$, pink plot $=\mathrm{T} 6$, grey plot $=\mathrm{T} 7$, green $\mathrm{plot}=$ control, and red line $=$ threshold line . 
combination of biological SNPs with imipenem only to Pantoea sp. 1, 2 (Appendix 10-13), respectively. T-test analysis comparing the combination of chemical silver NPs with antibiotics and biological silver NPs with antibiotics showed no significant difference as of $p>0.005$ in all experimental biofilm bacterial isolates (Appendix 14-17) to S. fonticola 1, 2, S. fonticola 3, Pantoea sp. 1, 2, and Pantoea sp. 3, 4, respectively.

\section{Conclusions}

In comparison with chemical synthesized silver NPs, biological silver NPs had more potent antimicrobial effect on biofilm bacterial isolates. In addition to the synergistic effect of silver NPs and antibiotics as a results of combining them, their effects on biofilm forming bacteria and gene expression of biofilm encoding genes (smaI and esaL) were down regulated when treated with silver NPs in both types, and increased when treated with the combination of silver NPs and antibiotics. But the most effective among them was biological silver NPs alone and in combination with antibiotics.

\section{References}

[1] H. Klasen, A historical review of the use of silver in the treatment of burns. II. Renewed interest for silver. Burns, 2000, 26(2): 131-138.

[2] B.S. Atiyeh, Effect of silver on burn wound infection control and healing: review of the literature. Burns, 2007. 33(2): 139-148.

[3] L. Sintubin, Lactic acid bacteria as reducing and capping agent for the fast and efficient production of silver nanoparticles. Applied Microbiology and Biotechnology, 2009, 84(4): 741-749.

[4] M.J. Al-Awady, G.M. Greenway, and V.N. Paunov, Nanotoxicity of polyelectrolyte-functionalized titania nanoparticles towards microalgae and yeast: role of the particle concentration, size and surface charge. RSC Advances, 2015, 5(46): 37044-37059.

[5] K.J. Kim, Antifungal activity and mode of action of silver nano-particles on Candida albicans. Biometals, 2009, 22(2): 235-242.

[6] P.L. Nadworny, Anti-inflammatory activity of nanocrystalline silver in a porcine contact dermatitis model. Nanomedicine: Nanotechnology, Biology and Medicine, 2008, 4(3): 241-251.

[7] J.V. Rogers, A preliminary assessment of silver nanoparticle inhibition of monkeypox virus plaque formation. Nanoscale Research Letters, 2008. 3(4): 129.

[8] S. Gurunathan, Antiangiogenic properties of silver nanoparticles. Biomaterials, 2009, 30(31): 6341-6350.

[9] F. Furno, Silver nanoparticles and polymeric medical devices: a new approach to prevention of infection? Journal of Antimicrobial Chemotherapy, 2004, 54(6): 1019-1024.

[10] K.N. Stevens, Hydrophilic surface coatings with embedded biocidal silver nanoparticles and sodium heparin for central venous catheters. Biomaterials, 2011, 32(5): 1264-1269.

[11] P. Li, Synergistic antibacterial effects of $\beta$-lactam antibiotic combined with silver nanoparticles. Nanotechnology, 2005, 16(9): 1912.

[12] S. Ruden, Synergistic interaction between silver nanoparticles and membrane-permeabilizing antimicrobial peptides. Antimicrobial Agents and Chemotherapy, 2009, 53(8): 3538-3540.

[13] I.S. Hwang, Synergistic effects between silver nanoparticles and antibiotics and the mechanisms involved. Journal of Medical Microbiology, 2012, 61(12): 1719-1726.

[14] S. Lynch, Role of the rapA gene in controlling antibiotic resistance of Escherichia coli biofilms. Antimicrobial Agents and Chemotherapy, 2007, 51(10): 3650-3658.

[15] G.L. Archer, Staphylococcus aureus: A well-armed pathogen. Reviews of Infectious Diseases, 1998. 26(5): 1179-1181.

[16] E.T. Hwang, Analysis of the toxic mode of action of silver nanoparticles using stress - specific bioluminescent bacteria. Small, 2008, 4(6): 746-750.

[17] W.K. Jung, Antibacterial activity and mechanism of action of the silver ion in Staphylococcus aureus and Escherichia coli. Appl. Environ. Microbiol, 2008, 74(7): 2171-2178.

[18] I.H. Al-Azawi, A.H. Al-Hamadani, and S.O. Hasson, Association between Biofilm Formation and Susceptibility to Antibiotics in Staphylococcus Lentus Isolated from Urinary Catheterized Patients. Nano Biomed. Eng, 2018. 10(2): 97-103.

[19] S.O. Hasson, A.H. Al-Hamadani, and I.H. Al-Azawi, Occurrence of biofilm formation in Serratia fonticola and Pantoea sp. isolates among urinary catheterized patients. Nano Biomed. Eng, 2018. 10(3): 295-304.

[20] M.U. Rashid, M.K.H. Bhuiyan, and M.E. Quayum, Synthesis of silver nano particles (Ag-NPs) and their uses for quantitative analysis of vitamin C tablets. Dhaka University Journal of Pharmaceutical Sciences, 2013. 12(1): 29-33.

[21] A.R. Shahverdi, Rapid synthesis of silver nanoparticles using culture supernatants of Enterobacteria: A novel biological approach. Process Biochemistry, 2007, 42(5): 919-923.

[22] B.A. Forbes, D.F. Sahm, and A.S. Weissfeld, Bailey \& Scott's diagnostic microbiology. Mosby, 2007.

[23] C. Wang, Green synthesis of silver nanoparticles by Bacillus methylotrophicus, and their antimicrobial activity. Artificial Cells, Nanomedicine, and Biotechnology, 2016, 44(4): 1127-1132.

[24] B. Kumar, Fabrication of silver nanoplates using Nephelium lappaceum (Rambutan) peel: A sustainable approach. Journal of Molecular Liquids, 2015, 211: 476480.

[25] F.P. Mehr, M. Khanjani, and P. Vatani, Synthesis of nanoAg particles using sodium borohydride. Oriental Journal of Chemistry, 2015, 31(3): 1831-1833.

[26] S. Gurunathan, Enhanced antibacterial and anti-biofilm activities of silver nanoparticles against Gram-negative and Gram-positive bacteria. Nanoscale Research Letters, 2014, 9(1): 373.

[27] V.R. Pasupuleti, Biogenic silver nanoparticles using Rhinacanthus nasutus leaf extract: synthesis, spectral analysis, and antimicrobial studies. International Journal of Nanomedicine, 2013, 8: 3355.

[28] R. Ghotaslou, The in vitro effects of silver nanoparticles on bacterial biofilms. The Journal of Microbiology, Biotechnology and Food Sciences, 2017, 6(4): 1077.

[29] G. Martinez-Castanon, Synthesis and antibacterial activity of silver nanoparticles with different sizes. Journal of Nanoparticle Research, 2008, 10(8): 1343-1348. 
[30] K. Chaloupka, Y. Malam, and A.M. Seifalian, Nanosilver as a new generation of nanoproduct in biomedical applications. Trends in Biotechnology, 2010, 28(11): 580588.

[31] A. Gade, Mycogenic metal nanoparticles: progress and applications. Biotechnology Letters, 2010, 32(5): 593600.

[32] T. Mathur, Detection of biofilm formation among the clinical isolates of staphylococci: an evaluation of three different screening methods. Indian Journal of Medical Microbiology, 2006, 24(1): 25.

[33] M.G. Guzmán, J. Dille, and S. Godet, Synthesis of silver nanoparticles by chemical reduction method and their antibacterial activity. Int J Chem Biomol Eng, 2009, 2(3): 104-111.

[34] M.A. Ansari, H.M. Khan, A.A. Khan, et al., Evaluation of antibacterial activity of silver nanoparticles against MSSA and MSRA on isolates from skin infections. Biology and Medicine, 2011, 3(2 Special Issue): 141-146.

[35] M.A. Shaker, M.I. Shaaban, Synthesis of silver nanoparticles with antimicrobial and anti-adherence activities against multidrug-resistant isolates from Acinetobacter baumannii. Journal of Taibah University Medical Sciences, 2017, 12(4): 291-297.

[36] D.K. Tiwari, J. Behari, and P. Sen, Time and dosedependent antimicrobial potential of Ag nanoparticles synthesized by top-down approach. Current Science, 2008: 647-655.

[37] J.D. Siegel, 2007 guideline for isolation precautions: preventing transmission of infectious agents in health care settings. American Journal of Infection Control, 2007, 35(10): S65-S164.

[38] D. Paredes, C. Ortiz, and R. Torres, Synthesis, characterization, and evaluation of antibacterial effect of Ag nanoparticles against Escherichia coli O157: H7 and methicillin-resistant Staphylococcus aureus (MRSA). International Journal of Nanomedicine, 2014, 9: 1717.

[39] L. Kvitek, Effect of surfactants and polymers on stability and antibacterial activity of silver nanoparticles (NPs). The Journal of Physical Chemistry C, 2008, 112(15): 5825-5834.

[40] J.R. Morones, The bactericidal effect of silver nanoparticles. Nanotechnology, 2005, 16(10): 2346.

[41] M. Marini, Antibacterial activity of plastics coated with silver-doped organic-inorganic hybrid coatings prepared by sol-gel processes. Biomacromolecules, 2007, 8(4): 1246-1254.

[42] K.B. Holt, A.J. Bard, Interaction of silver (I) ions with the respiratory chain of Escherichia coli: an electrochemical and scanning electrochemical microscopy study of the antimicrobial mechanism of micromolar $\mathrm{Ag}^{+}$. Biochemistry, 2005, 44(39): 13214-13223.
[43] S. Gurunathan, Rapid biological synthesis of silver nanoparticles and their enhanced antibacterial effects against Escherichia fergusonii and Streptococcus mutans. Arabian Journal of Chemistry, 2019, 12(2): 168-180.

[44] S.K.R. Namasivayam, Biofilm inhibitory effect of silver nanoparticles coated catheter against Staphylococcus aureus and evaluation of its synergistic effects with antibiotics. Int J Biol Pharm Res, 2012, 3(2): 259-265.

[45] V. Kostenko, Impact of silver-containing wound dressings on bacterial biofilm viability and susceptibility to antibiotics during prolonged treatment. Antimicrobial Agents and Chemotherapy, 2010, 54(12): 5120-5131.

[46] H. Tan, The use of quaternised chitosan-loaded PMMA to inhibit biofilm formation and downregulate the virulenceassociated gene expression of antibiotic-resistant staphylococcus. Biomaterials, 2012, 33(2): 365-377.

[47] J. Hellemans, J. Vandesompele, Quantitative real-time PCR: Methods and protocols. Methods in Molecular Biology, 2014, 1160: 19-27.

[48] K.J. Livak, T.D. Schmittgen, Analysis of relative gene expression data using real-time quantitative PCR and the 2- $\Delta \Delta$ CT method. Methods, 2001, 25(4): 402-408.

[49] H.-B. Li, Screening potential reference genes for quantitative real-time PCR analysis in the oriental armyworm, Mythimna separata. PloS One, 2018, 13(4): e0195096.

[50] W.J. Fu, Statistical models in assessing fold change of gene expression in real-time RT-PCR experiments. Computational Biology and Chemistry, 2006, 30(1): 2126.

[51] T.D. Schmittgen, K.J. Livak, Analyzing real-time PCR data by the comparative C T method. Nature Protocols, 2008, 3(6): 1101.

[52] F. Liu, Identification of reference genes for gene expression normalization in safflower (Carthamus tinctorius). Revista Brasileira de Farmacognosia, 2016, 26(5): 564-570.

[53] A.J. Wilkinson, Biofilm formation and antibiotic resistance on alginate beads, of Staphylococcus aureus and other health care associated bacterial species. Thesis presented for the Degree of Doctor of Philosophy, The University of Edinburgh, 2016.

Copyright $(\subset$ Shaimaa Obaid Hasson, Mohammed Jabber AlAwady, Adnan Hamad Al-Hamadani, Ibtisam Habeeb Al-Azawi, and Alaa Irhayyim Ali. This is an open-access article distributed under the terms of the Creative Commons Attribution License, which permits unrestricted use, distribution, and reproduction in any medium, provided the original author and source are credited. 